\title{
A Half-Inverse Problem for Impulsive Dirac Operator with Discontinuous Coefficient
}

\author{
Yalçın Güldü \\ Cumhuriyet University, Faculty of Science, Department of Mathematics, 58140 Sivas, Turkey \\ Correspondence should be addressed to Yalçın Güldü; yguldu@gmail.com
}

Received 3 June 2013; Accepted 9 September 2013

Academic Editor: Agacik Zafer

Copyright (C) 2013 Yalçın Güldü. This is an open access article distributed under the Creative Commons Attribution License, which permits unrestricted use, distribution, and reproduction in any medium, provided the original work is properly cited.

An inverse problem for Dirac differential operators with discontinuity conditions and discontinuous coefficient is studied. It is shown by Hochstadt and Lieberman's method that if the potential function $p(x)$ in $\Omega(x)$ is prescribed over the interval $(\pi / 2, \pi)$, then a single spectrum suffices to determine $p(x)$ on the interval $(0, \pi)$ and it is also shown here that $\rho(x)$ is uniquely determined by a spectrum.

\section{Introduction}

In this paper, we are concerned with the Dirac operator $L$ generated by equation

$$
\begin{array}{r}
\ell(y):=B y^{\prime}(x)+\Omega(x) y(x)=\lambda \rho(x) y(x), \\
x \in I:=\left(0, \frac{\pi}{2}\right) \cup\left(\frac{\pi}{2}, \pi\right)
\end{array}
$$

with

$$
\begin{aligned}
& B=\left(\begin{array}{cc}
0 & 1 \\
-1 & 0
\end{array}\right), \quad \Omega(x)=\left(\begin{array}{cc}
p(x) & q(x) \\
q(x) & -p(x)
\end{array}\right), \\
& \rho(x)= \begin{cases}1, & 0 \leq x<\frac{\pi}{2} \\
\alpha, & \frac{\pi}{2}<x \leq \pi,\end{cases}
\end{aligned}
$$

where $1<\alpha \in \mathbb{R}^{+}, \quad y(x)=\left(\begin{array}{l}y_{1}(x) \\ y_{2}(x)\end{array}\right)$, subject to the boundary conditions

$$
\begin{aligned}
& y_{1}(0)=0, \\
& y_{2}(\pi)=0,
\end{aligned}
$$

and discontinuity conditions

$$
y\left(\frac{\pi}{2}+0\right)=A y\left(\frac{\pi}{2}-0\right),
$$

where $p(x)$ and $q(x)$ are real valued functions in $L_{2}(0, \pi), \lambda$ is a spectral parameter, and $A=\left(\begin{array}{cc}\beta & 0 \\ 0 & \beta^{-1}\end{array}\right), \beta \in \mathbb{R}^{+} \backslash\{1\}$.

Inverse problems of spectral analysis implicate the reconstruction of a linear operator from its spectral data (e.g., see [1-5]). Half inverse problem for a Dirac operator consists in reconstruction of this operator from its spectrum and half of the potential.

The first result on the half-inverse problem is due to Hochstadt and Lieberman [6]. After that, Hald [7] proved that if the potential is known over half of the interval and if one boundary conditions is given, then the potential and the other boundary condition are uniquely determined by the eigenvalues. In $[8,9]$, Malamud and Gesztesy and Simon obtained some new uniqueness results in inverse spectral analysis with partial information on the potential for scalar and matrix Sturm-Liouville equations, respectively. In 2001, Sakhnovich [10] studied the existence of solution to the halfinverse problem. In [11], necessary and sufficient condition for solvability of the half-inverse spectral problem for SturmLiouville operators with singular operator was taken. In [12], by using the Hochstadt and Lieberman's method, halfinverse problem was solved for diffusion operators. In [13], the authors presented half-inverse problem for the SturmLiouville equation with eigenparameter-dependent boundary conditions.

On the other hand, the fundamental and detailed results about Dirac operators were given in [14]. Moreover, direct or 
inverse spectral problems for Dirac operators are largely well studied in $[1,8,15]$.

There are also some studies about the interior inverse problems. Arutyunyan [16] proved that the eigenvalues $\lambda_{n}$, $n=0,1, \ldots$ and normalizing coefficients $\alpha_{n}=\left\|y_{n}\right\|_{\left\{L^{2}(0.1)\right\}^{2}}$, $n=0,1, \ldots$ uniquely determined the potential $Q(x)$. Malamud [8] proved an analog of Borg theorem [17]; he showed that the spectra of two boundary value problems for an operator with different boundary conditions at one end uniquely determined the potential $Q(x)$. He also proved an analog of the theorem of Hochstadt and Lieberman [6]; one spectrum and a potential on the interval $(0,1 / 2)$ uniquely determined the potential $Q(x)$ on the whole interval $[0,1]$. On the other hand, in 2001, Del Rio and Grébert [18] proved that in the case where $\varphi$ is a priori known on $[a, 1]$, then only a part (depending on $a$ ) of two spectra determined $\varphi$ on $[0,1]$. Furthermore, inverse problems for interior spectral data of the Sturm-Liouville and Dirac operators were studied in [1922].

The jump conditions like (5) appear in-some important physical problems. The work in [7] is a well-known work about discontinuous inverse eigenvalue problems. Direct and inverse problems for Dirac operators with discontinuities inside the interval were investigated in [23].

In this paper, by using the Hochstadt and Lieberman's method in [6], we discuss the half-inverse problem for Dirac operator with discontinuity conditions and discontinuous coefficients (1)-(5). Furthermore, the potential $p(x)$ and discontinuous coefficient $\rho(x)$ are uniquely determined.

\section{Statement of Results}

Let the function $\varphi(\cdot, \lambda): I \rightarrow R^{2}$ be solution of (1) which satisfies the initial conditions

$$
\varphi(0, \lambda)=\left(\begin{array}{c}
0 \\
-1
\end{array}\right)
$$

and the jump conditions (5).

It is shown in [14] that, for the solution $\varphi(x, \lambda)$, the following representation holds:

$$
\varphi(x, \lambda)=\varphi_{0}(x, \lambda)+\int_{0}^{x} K(x, t) \varphi_{0}(t, \lambda) d t, \quad \text { for } 0<x<\frac{\pi}{2},
$$

where $\varphi_{0}(x, \lambda)=\left(\varphi_{01}(x, \lambda), \varphi_{02}(x, \lambda)\right)^{T}$ has the form

$$
\begin{gathered}
\varphi_{01}(x, \lambda)= \begin{cases}\sin \lambda x, & 0 \leq x<\frac{\pi}{2} \\
\beta^{+} \sin \lambda \mu(x) & \\
+\beta^{-} \sin \lambda(\pi-\mu(x)), & \frac{\pi}{2}<x \leq \pi,\end{cases} \\
\varphi_{02}(x, \lambda)=\left\{\begin{array}{cc}
-\cos \lambda x, & 0 \leq x<\frac{\pi}{2} \\
-\beta^{+} \cos \lambda \mu(x) & \frac{\pi}{2}<x \leq \pi, \\
+\beta^{-} \cos \lambda(\pi-\mu(x)), &
\end{array}\right.
\end{gathered}
$$

$\beta^{ \pm}=(1 / 2)(\beta \pm \beta), K(x, t)=\left(K_{i j}(x, t)\right)_{i, j=1,2}$, and $K_{i j}(x, t)$ are real valued continuous functions for $i, j=1,2$ and for each

$$
x, \mu(x)= \begin{cases}x, & 0 \leq x<\pi / 2 \\ \alpha x-\alpha(\pi / 2)+(\pi / 2), & \pi / 2<x \leq \pi\end{cases}
$$

Next, we define the function

$$
\Delta(\lambda)=\varphi_{2}(\pi, \lambda)
$$

The zeros of $\Delta(\lambda)$ which is called the characteristic function of the problem (1)-(5) are the eigenvalues of $L$.

From the equalities (7)-(11), we have

$$
\Delta(\lambda)=\Delta_{0}(\lambda)+o(\exp \tau \mu(\pi))
$$

where $\Delta_{0}(\lambda)=-\beta^{+} \cos \lambda \mu(\pi)+\beta^{-} \cos \lambda(\pi-\mu(\pi))$ and $\tau=$ $|\operatorname{Im} \lambda|$.

Theorem 1. (i) The problem L has denumerable many eigenvalues such that all of them are real and simple.

(ii) The eigenvalues $\lambda_{n}$ are expressed by the following asymptotic formula:

$$
\lambda_{n}=\lambda_{n}^{0}+O(1)
$$

where $\lambda_{n}^{0}$ are the zeros of $\Delta_{0}(\lambda)$ and $\lambda_{n}^{0}=n \pi / \mu(\pi)+h_{n}$, $\sup _{n}\left|h_{n}\right|<\infty$.

Proof. (i) Since $\Delta(\lambda)$ is entire function, it has denumerable many zeros. Moreover, from [23], zeros of $\left\{\lambda_{n}\right\}$ are real and simple.

(ii) It is shown in [24] that $\lambda_{n}^{0}=n \pi / \mu(\pi)+O(1)$. It is obvious that $\left|\Delta_{0}(\lambda)\right| \geq C_{\delta} \exp \tau \mu(\pi)$ for $\lambda \in G_{\delta}:=\{\lambda: \mid \lambda-$ $\left.\lambda_{n} \mid>\delta\right\}$ and $\Delta(\lambda)-\Delta_{0}(\lambda)=o(\exp \tau \mu(\pi))$.

Therefore, it follows from the Rouche's theorem that the functions $\Delta_{0}(\lambda)$ and $\Delta(\lambda)$ have the same number of zeros inside the contour $\gamma_{\varepsilon}:=\left\{\lambda:|\lambda|=\left|\lambda_{n}^{0}\right|-\varepsilon\right\}$; that is, the eigenvalues $\lambda_{n}$ are given by the following asymptotic formula:

$$
\lambda_{n}=\frac{n \pi}{\mu(\pi)}\left(1+O\left(\frac{1}{n}\right)\right) \text {. }
$$

Consider a second operator $\widetilde{L}$ generated by the differential equation

$$
\begin{array}{r}
B y^{\prime}(x)+\widetilde{\Omega}(x) y(x)=\lambda \widetilde{\rho}(x) y(x), \\
x \in I:=\left(0, \frac{\pi}{2}\right) \cup\left(\frac{\pi}{2}, \pi\right)
\end{array}
$$

subject to the same boundary conditions (3) and (4) and discontinuity condition (5). Here, $\widetilde{\Omega}(x)=\left(\begin{array}{cc}\widetilde{p}(x) & q(x) \\ q(x) & -\widetilde{p}(x)\end{array}\right)$ with a real valued function $\widetilde{p}(x) \in L_{2}(0, \pi)$.

We denote eigenvalues by $\lambda_{n}$ and the corresponding eigenfunctions by $\varphi_{n}(x)=\varphi\left(x, \lambda_{n}\right)$ of the problem $L$ and denote eigenvalues by $\widetilde{\lambda}_{n}$ and the corresponding eigenfunctions by $\widetilde{\varphi}_{n}(x)=\varphi\left(x, \tilde{\lambda}_{n}\right)$ of the problem $\widetilde{L}$. 
Lemma 2. If $\lambda_{n}=\widetilde{\lambda}_{n}$, then $\alpha=\tilde{\alpha}$ that is, $\rho(x)=\tilde{\rho}(x)$.

Proof. Since $\lambda_{n}=(n \pi / \mu(\pi))(1+O(1 / n))$, then $\tilde{\lambda}_{n}=$ $(n \pi / \widetilde{\mu}(\pi))(1+O(1 / n)),(n \pi / \mu(\pi))(1+O(1 / n))=(n \pi / \widetilde{\mu}(\pi))(1+$ $O(1 / n))$. Letting $n \rightarrow \infty$, then we conclude that $\mu(\pi)=$ $\widetilde{\mu}(\pi)$. Moreover, since $\mu(\pi)=(\pi / 2)(\alpha+1)$, then $\alpha=\widetilde{\alpha}$. So $\rho(x)=\tilde{\rho}(x)$.

Theorem 3. If $\lambda_{n}=\widetilde{\lambda}_{n}$, for all $n \in \mathbb{N}$ and $p(x)=\widetilde{p}(x)$, for $x \in(\pi / 2, \pi)$, then $p(x)=\widetilde{p}(x)$ almost everywhere on $(0, \pi)$.

Proof. Let us write (1) for the solutions $\varphi$ and $\widetilde{\varphi}$ and take into account Lemma 2 as

$$
\begin{aligned}
& B \varphi^{\prime}(x, \lambda)+\Omega(x) \varphi(x, \lambda)=\lambda \rho(x) \varphi(x, \lambda), \\
& B \widetilde{\varphi}^{\prime}(x, \lambda)+\widetilde{\Omega}(x) \widetilde{\varphi}(x, \lambda)=\lambda \rho(x) \widetilde{\varphi}(x, \lambda) .
\end{aligned}
$$

If we multiply these equalities by $\tilde{\varphi}(x, \lambda)$ and $\varphi(x, \lambda)$, respectively, and subtract, then we obtain

$$
\begin{gathered}
\frac{d}{d x}\left\{\varphi_{1}(x, \lambda) \widetilde{\varphi}_{2}(x, \lambda)-\widetilde{\varphi}_{1}(x, \lambda) \varphi_{2}(x, \lambda)\right\} \\
=[\Omega(x)-\widetilde{\Omega}(x)] \varphi(x, \lambda) \widetilde{\varphi}(x, \lambda) .
\end{gathered}
$$

Integrating the last equality from 0 to $\pi$ with respect to $x$, the equality

$$
\begin{aligned}
& \left.\left\{\varphi_{1}(x, \lambda) \widetilde{\varphi}_{2}(x, \lambda)-\widetilde{\varphi}_{1}(x, \lambda) \varphi_{2}(x, \lambda)\right\}\right|_{0} ^{\pi} \\
& =\int_{0}^{\pi}[\Omega(x)-\widetilde{\Omega}(x)] \varphi(x, \lambda) \widetilde{\varphi}(x, \lambda) d x \\
& =\int_{0}^{\pi}[p(x)-\widetilde{p}(x)] J \varphi(x, \lambda) \widetilde{\varphi}(x, \lambda) d x
\end{aligned}
$$

is obtained where $J:=\left(\begin{array}{cc}1 & 0 \\ 0 & -1\end{array}\right)$. Applying the initial condition (6) and the assumption $p(x)=\widetilde{p}(x), x \in(\pi / 2, \pi)$ in hypothesis, we get

$$
\begin{aligned}
& \left.\left\{\varphi_{1}(x, \lambda) \widetilde{\varphi}_{2}(x, \lambda)-\widetilde{\varphi}_{1}(x, \lambda) \varphi_{2}(x, \lambda)\right\}\right|_{0} ^{\pi} \\
& \quad=\int_{0}^{\pi / 2}[\Omega(x)-\widetilde{\Omega}(x)] \varphi(x, \lambda) \widetilde{\varphi}(x, \lambda) d x \\
& \quad=\int_{0}^{\pi / 2}[p(x)-\tilde{p}(x)] J \varphi(x, \lambda) \tilde{\varphi}(x, \lambda) d x .
\end{aligned}
$$

Define

$$
F(\lambda):=\int_{0}^{\pi / 2}[p(x)-\tilde{p}(x)] J \varphi(x, \lambda) \widetilde{\varphi}(x, \lambda) d x
$$

where

$$
\begin{aligned}
J \varphi(x, \lambda) \tilde{\varphi}(x, \lambda)= & -\cos 2 \lambda x+\int_{0}^{x} K_{1}(x, t) \cos 2 \lambda t d t \\
& +\int_{0}^{x} K_{2}(x, t) \sin 2 \lambda t d t
\end{aligned}
$$

and $K_{i}(x, t), i=1,2$ depend only on $x, t$.
Then we get from the boundary condition (4) that

$$
F\left(\lambda_{n}\right)=0
$$

for all $n$.

Now, define

$$
\chi(\lambda):=\frac{F(\lambda)}{\Delta(\lambda)}
$$

$\chi(\lambda)$ is an entire function. Since $F(\lambda)=O(\exp \tau \pi)$ and $|\Delta(\lambda)| \geq C_{\delta} \exp \tau \mu(\pi)$ for $\lambda \in G_{\delta}:=\left\{\lambda:\left|\lambda-\lambda_{n}\right|>\delta\right\}$ where $\mu(\pi)=(\pi / 2)(\alpha+1)$, then $\chi(\lambda)$ is constant from the Liouville's theorem. Furthermore,

$$
\lim _{\lambda \rightarrow \infty} \chi(\lambda)=0
$$

from the equalities (7), (9), and (21) and the RiemannLebesgue lemma. Thus, $\chi(\lambda)=0$ on the whole $\lambda$-plane.

It follows from (20) and (21) that

$$
\begin{array}{r}
\int_{0}^{\pi / 2} Q(x)\left\{\cos 2 \lambda x-\int_{0}^{x} K_{1}(x, t) \cos 2 \lambda t d t\right. \\
\left.-\int_{0}^{x} K_{2}(x, t) \sin 2 \lambda t d t\right\} d x=0
\end{array}
$$

for all $\lambda$ where $Q(x):=[p(x)-\widetilde{p}(x)]$. This can be rewritten as

$$
\begin{array}{r}
\int_{0}^{\pi / 2} \cos 2 \lambda \tau\left[Q(\tau)+\int_{\tau}^{\pi / 2} Q(x) K_{1}(x, t) d x\right] d t \\
+\int_{0}^{\pi / 2} \sin 2 \lambda t \int_{\tau}^{\pi / 2} Q(x) K_{2}(x, t) d x d t=0 .
\end{array}
$$

From the completeness of the functions $(\cos 2 \lambda \tau$, $\sin 2 \lambda t)^{T}$ in $L_{2}(0, \pi) \oplus L_{2}(0, \pi)$, we have

$$
Q(\tau)+\int_{\tau}^{\pi / 2} Q(x) K_{1}(x, t) d x=0, \quad \text { for } 0<\tau<\frac{\pi}{2} .
$$

It follows that $Q(x)=0$; that is, $p(x)=\widetilde{p}(x)$ almost everywhere for $x \in(0, \pi)$.

\section{References}

[1] I. M. Gelfand and B. M. Levitan, "On the determination of a differential equation from its spectral function," Izvestiya Akademii Nauk SSSR, vol. 15, no. 4, pp. 309-360, 1951.

[2] B. M. Levitan, "On the determination of the Sturm-Liouville operator from one and two spectra," Mathematics of the USSRIzvestiya, vol. 12, pp. 179-193, 1978.

[3] B. M. Levitan, Inverse Sturm-Liouville Problems, VNU Science Press, Utrecht, The Netherlands, 1987.

[4] J. Pöschel and E. Trubowitz, Inverse Spectral Theory, Academic Press, Orlando, Fla, USA, 1987.

[5] D. Alpay and I. Gohberg, "Inverse problems associated to a canonical differential system," in Recent Advances in Operator Theory and Related Topics, vol. 127 of Operator Theory: Advances and Applications, pp. 1-27, 2001. 
[6] H. Hochstadt and B. Lieberman, "An inverse Sturm-Liouville problem with mixed given data," SIAM Journal on Applied Mathematics, vol. 34, no. 4, pp. 676-680, 1978.

[7] O. H. Hald, "Discontinuous inverse eigenvalue problems," Communications on Pure and Applied Mathematics, vol. 37, no. 5, pp. 539-577, 1984.

[8] M. M. Malamud, "Uniqueness questions in inverse problems for systems of differential equations on a finite interval," Transactions of the Moscow Mathematical Society, vol. 60, pp. 204-262, 1999.

[9] F. Gesztesy and B. Simon, "Inverse spectral analysis with partial information on the potential. II. The case of discrete spectrum," Transactions of the American Mathematical Society, vol. 352, no. 6, pp. 2765-2787, 2000.

[10] L. Sakhnovich, "Half-inverse problems on the finite interval," Inverse Problems, vol. 17, no. 3, pp. 527-532, 2001.

[11] R. O. Hryniv and Y. V. Mykytyuk, "Half-inverse spectral problems for Sturm-Liouville operators with singular potentials," Inverse Problems, vol. 20, no. 5, pp. 1423-1444, 2004.

[12] H. Koyunbakan and E. S. Panakhov, "Half-inverse problem for diffusion operators on the finite interval," Journal of Mathematical Analysis and Applications, vol.326, no. 2, pp. 1024-1030, 2007.

[13] C.-F. Yang and Z.-Y. Huang, "A half-inverse problem with eigenparameter dependent boundary conditions," Numerical Functional Analysis and Optimization, vol. 31, no. 4-6, pp. 754762, 2010.

[14] B. M. Levitan and I. S. Sargsjan, Sturm-Liouville and Dirac Operators, Kluwer Academic Publishers, Dordrecht, The Netherlands, 1991.

[15] M. G. Gasymov and B. M. Levitan, "The inverse problem for the Dirac system," Doklady Akademii Nauk SSSR, vol. 167, pp. 967-970, 1966.

[16] T. N. Arutyunyan, "Isospectral Dirac operators," Izvestiya Akademii Nauk Armenii. Matematika, vol. 29, no. 2, pp. 3-14, 1994.

[17] G. Borg, "Eine umkehrung der sturm-liouvilleschen eigenwertaufgabe, bestimmung der Differential gleichung durch die eigenvert," Acta Mathematica, vol. 78, pp. 1-96, 1946.

[18] R. del Rio and B. Grébert, "Inverse spectral results for AKNS systems with partial information on the potentials," Mathematical Physics, Analysis and Geometry, vol. 4, no. 3, pp. 229-244, 2001.

[19] K. Mochizuki and I. Trooshin, "Inverse problem for interior spectral data of the Sturm-Liouville operator," Journal of Inverse and Ill-Posed Problems, vol. 9, no. 4, pp. 425-433, 2001.

[20] K. Mochizuki and I. Trooshin, "Inverse problem for interior spectral data of the Dirac operator on a finite interval," Kyoto University. Research Institute for Mathematical Sciences, vol. 38, no. 2, pp. 387-395, 2002.

[21] C.-F. Yang and X.-P. Yang, "An interior inverse problem for the Sturm-Liouville operator with discontinuous conditions," Applied Mathematics Letters, vol. 22, no. 9, pp. 1315-1319, 2009.

[22] A. S. Ozkan and R. Kh. Amirov, "An interior inverse problem for the impulsive Dirac operator," Tamkang Journal of Mathematics, vol. 42, no. 3, pp. 259-263, 2011.

[23] R. Kh. Amirov, "On a system of Dirac differential equations with discontinuity conditions inside an interval," Ukrainian Mathematical Journal, vol. 57, no. 5, pp. 712-727, 2005.

[24] V. F. Zhdanovich, "Formulas for the zeros of Dirichlet polynomials and quasipolynomials," Doklady Akademii Nauk SSSR, vol. 13, no. 5, pp. 1046-1049, 1960. 


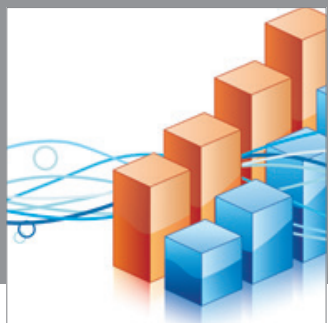

Advances in

Operations Research

mansans

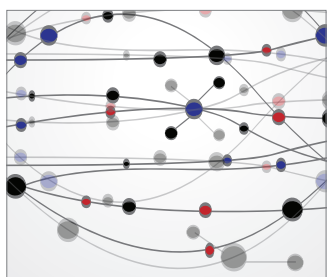

The Scientific World Journal
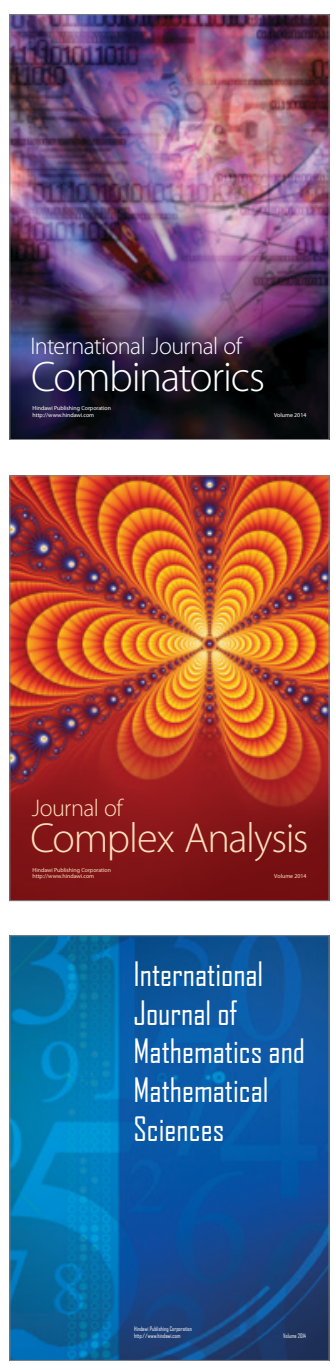
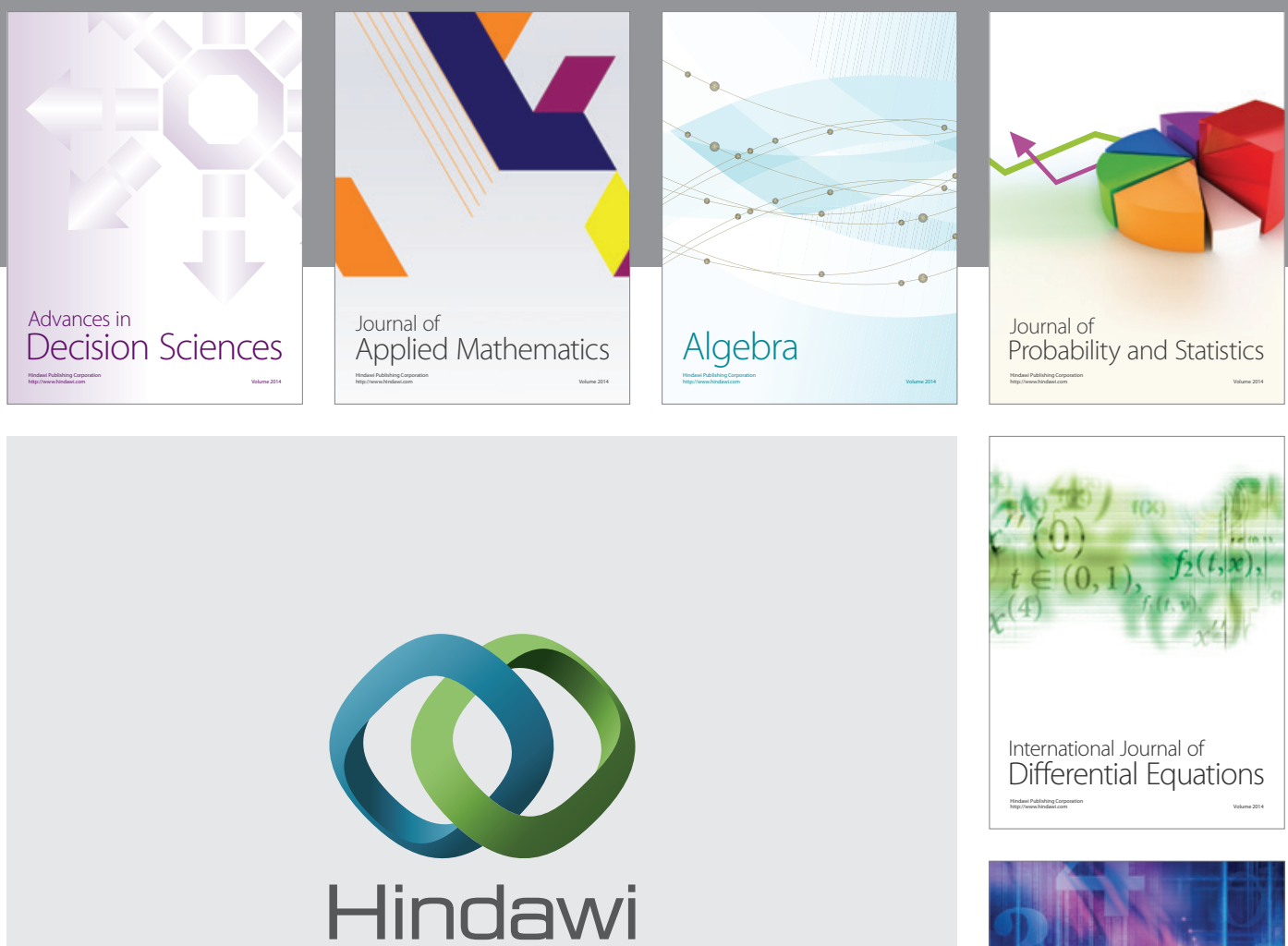

Submit your manuscripts at http://www.hindawi.com
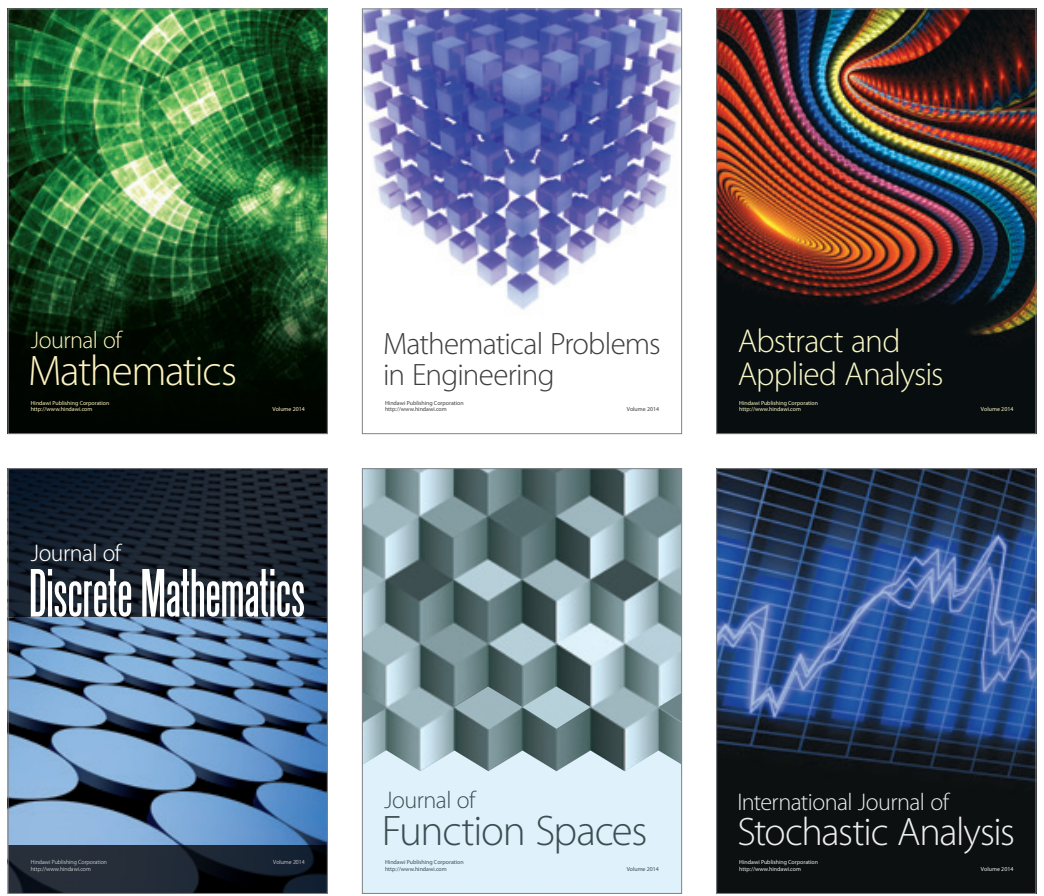

Journal of

Function Spaces

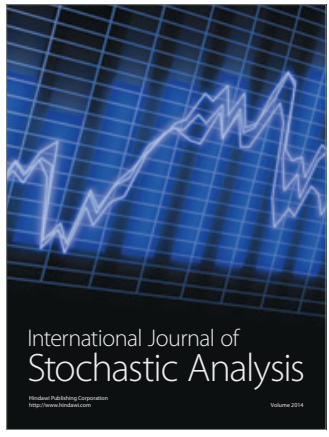

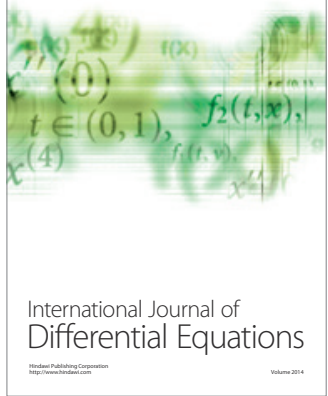
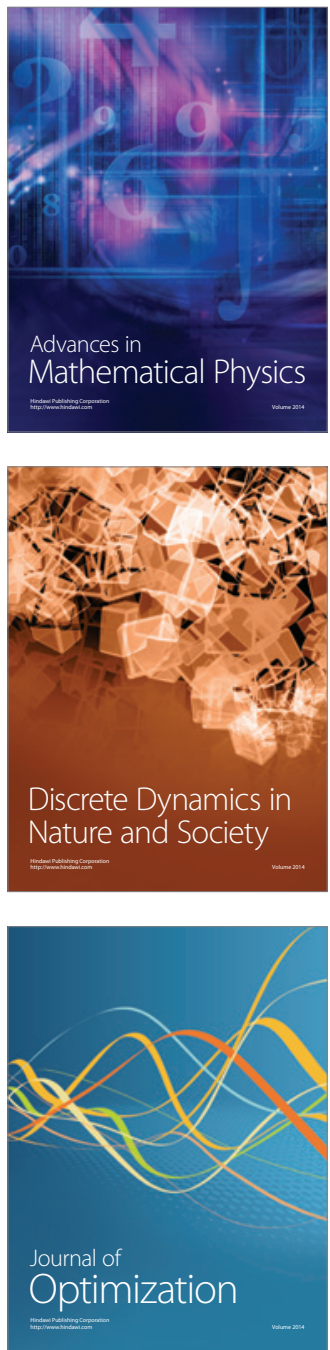